features of Crohn's on histology, whilst the remaining 27 had mildly active ileitis on histology. 22 of these 27 patients underwent additional investigations to confirm (19/22) or exclude IBD (3/22). 5 of the 27 patients with histological ileitis that was not typical of CD were diagnosed with IBD, without further evidence to demonstrate this. In total 35/38 (92.1\%) patients with histological and endoscopic evidence of inflammation were diagnosed with CD. Of the $18 / 56$ patients (32\%) with TI on endoscopy but normal histology, 9 had further investigations including MRI, barium studies and US abdomen to exclude CD. 4 patients were diagnosed with $\mathrm{CD}$ despite normal histology. 6/18 patients with normal histology were lost to follow up, although all patients diagnosed with IBD were followed up in GI clinic.

Conclusion We conclude that although the majority of patients with TI on endoscopy have CD, 1/3 of our patients had no histological correlation of inflammation. $16 \%$ of patients with endoscopic TI with mildly active ilieits on histology had no further imaging to authenticate a diagnosis of $\mathrm{CD}$ and $8 \%$ of patients with normal histology with no further investigations were labelled as CD. The latter group of patients may be inaccurately labelled with a diagnosis of CD and along with this the potential stigma associated with a chronic ailment, risk of escalation therapy with immununosuppressives/biologics and occasionally unnecessary surgery.

Disclosure of Interest None Declared.

\section{PWE-104 THE FINANCIAL IMPACT OF A NURSE-LED IBD (INFLAMMATORY BOWEL DISEASE) TELEPHONE ADVICE SERVICE, IN A LARGE DISTRICT GENERAL HOSPITAL}

S Squires*, A Boal, G Naismith. NHS Greater Glasgow and Clyde Health Board, Paisley, UK

\subsection{6/gutjnl-2014-307263.364}

Introduction It has become increasingly recognised that outpatient management is more cost effective in IBD. ${ }^{1}$ IBD Standards (Revised 2013) recommend telephone advice for patients with regards to symptoms and medication management. This report attempts to quantify the net financial impact of this service at our hospital since it was introduced in August 2013.

Methods The Royal Alexandra Hospital in Paisley is a District General Hospital with a catchment population of $200,000^{2}$ with approximately 2500 IBD patients. Data relating to the use of the Telephone Advice service was prospectively recorded on a daily basis for a period of 5 months. We Documented reasons for calling and the likely action taken by the patient had the telephone advice line not been available. Cost savings based on alternative outcomes were made in accordance the Department of health figures. ${ }^{3}$

Results The mean calls per month was was 88 [IQR 24] (Mean calls which were deemed Non-IBD issues was, was 30 calls per month [IQR 8.0]) The mean cost of staffing the IBD advice line with an IBD Clinical Nurse Specialist was $£ 482.00$ per month [IQR 195.5]. The mean time spent on calls per month was $28.5 \mathrm{~h}$ [IQR 11.5]. Cost Savings over 5 months for avoidance of GP consultation was $£ 3408.00$. Savings for avoidance of a consultant appointment made over the 5 month period was $£ 27454.00$. Savings made from patients avoiding either an $\mathrm{A}+\mathrm{E}$ or Hospital Admission were $£ 540.00$ and $£ 11488.00$ respectively over the 5 month period. The net saving was $£ 42890.00$.

Conclusion A Nurse-Led telephone advice line appears to be a cost effective intervention. It may prevent patients from unnecessary hospital attendance. Savings can be made to both primary and secondary care. Overall, it appears that the advice line is providing a highly valuable service, not just in terms of accessible treatment decisions and guidance for patients, but cost savings when Specialist Nurse time is compared to General Practitioners, Consultants or hospital facilities.

\section{REFERENCES}

1 Park KT, Bass D. Inflammatory bowel diseaseattributable costs and costeffective strategies in the United States: A review. Inflammatory Bowel Diseases, 2011 Wiley Online Library

2 NHSGGC (National Health Service, Greater Glasgow and Clyde) - Consultation document 2006-2012 http://www.nhsggc.org.uk

3 Department of Health reference Costs 2011-2012 https://www.gov.uk

Disclosure of Interest None Declared.

\section{PWE-105 BIOLOGICAL WORLD OF IBD, IS IT ONLY SAFE IN TERTIARY CARE CENTRES?}

${ }^{1} \mathrm{~S}$ Kadir*, ${ }^{2} \mathrm{R}$ Campbell, ${ }^{2} \mathrm{~N}$ Ahluwalia. 'Gastroenterology, Manchester Royal Informary, UK; ${ }^{2}$ Gastroenterology, Stepping Hill Hospital, Manchester, UK

\subsection{6/gutjnl-2014-307263.365}

Introduction Inflammatory bowel disease (IBD) has a prevalence of 400 per 100,000 with approximately 240,000 patient with IBD in UK. It is triggered by a combination of environmental, genetic and immunoregulatory factors including a dysfunctional mucosal immune response.

In the last 15 years, the introduction of biologicals has dramatically changed the landscape with improving natural history and outcome of IBD. Many multi-centre trials have confirmed the efficacy of both Infliximab (IFX) and Adalimumab (ADA) in the management of IBD. These drugs are however not without side effects and need close monitoring in specialist IBD clinics. We share our experience from a large-sized DGH [>400,000 population] in Northwest England with a dedicated IBD service and experience in the use of biologicals in IBD patients since 2000.

Methods We interrogated and audited our IBD data base and collected all the patients who were or had been on biologicals. The data goes back to the year 2000 .

Results The basic demographics showed total of the 90 patients; $42 \%$ female. The mean age was 42.4 (female) vs. 39.3 yrs (male). Age at the time of diagnosis of IBD was $31.1 \mathrm{yrs}$ for females and 32.4 years for males. 53\% had crohn's disease and rest were UC. Patients with UC had higher body weight vs. crohn's disease with a mean of 76.1 vs. $67.6 \mathrm{~kg}$ respectively. $58 \%$ had Ileoceacal Crohn's of which $73 \%$ were males. $62.5 \%$ of females with UC had pan colitis vs. $42 \%$ males.

All patients were fully informed and consented prior to the initiation of biological therapy. $89 \%$ of the patients were on immunomodulators prior to biological therapy. $93 \%$ of the patients with UC were on infliximab with $5 \%$ on Adalulimab. 1 pt was on Basiliximab which was changed to infliximab. Those with crohn's disease, $83 \%$ were on Infliximab and $17 \%$ on Adalulimab. Infliximab was stopped in $30 \%$ due to either no further clinical need or change to Adalulimab and of these $6 \%$ due to adverse events. In the ADA group 2\% stopped due to lack of clinical inefficacy. The median duration of biologicals was 38.2 months, maximum duration 156 months.

Conclusion In the context of IBD, biological drugs in a dedicated IBD service are highly efficacious. In our centre, paucity of adverse event maybe due to our cohort of patient population with little background incidence of TB. In contrast to the UK IBD audit, we had more usage of biologicals in the context of UC, of which $50 \%$ had pan colitis. Our experience shows that a treating IBD effectively and 